\title{
Classification of EEG Signals in Depressed Patients
}

\author{
S. G. ERALDEMIR, Ü. KILIÇ, M. KELEŞ, M. E. DEMIRKOL, E. YILDIRIM, L. TAMAM
}

\begin{abstract}
Electroencephalography (EEG) are electrical signals that occur in every activity of the brain. Investigation of normal and abnormal changes that take place in the human brain using EEG signals is a widely used method in recent years. The World Health Organization (WHO) states that one of the most important health problems in today's society is depressive disorders. Nowadays, various scales are used in the diagnosis of depressive disorder in individuals. These scales are based on the declaration of the individual. In recent studies, EEG has been used as a biomarker for the diagnosis of depression. In this study, EEG signals from 30 patients with clinical depressive disorder have been recorded. EEG signals have been collected for 1 minute with eyes open and closed. The collected data have been divided into attributes by continuous wavelet transform which is used in many studies in processing non-stationary signals such as EEG. Obtained attributes have been classified with kNN classification method. As a result, it was observed that EEG signals, collected from subjects with depression while eyes are open and closed, can be classified with an accuracy of $91.30 \%$.
\end{abstract}

Index Terms-EEG, Depressive Disorders, kNN, Wavelet Transform

SERVER GÖKSEL ERALDEMIR, is with Depertment of Iskenderun Vocational School of Iskenderun Technical University, HATAY, Turkey, (sgoksel.eraldemir@iste.edu.tr).

iD https://orcid.org/0000-0003-0835-2601

ÜMIT KILIÇ, is with Department Faculty Engineering of Adana Alparslan Turkes of Science and Technology University, Adana,Turkey, (ukilic@atu.edu.tr)

iD https://orcid.org/0000-0001-8067-6024

MÜMINE KAYA KELEŞ, is with Department Faculty Engineering of Adana Alparslan Turkes of Science and Technology University, Adana,Turkey, (e-mail: mkaya@atu.edu.tr).

(iD https://orcid.org/0000-0001-8414-1713

MEHMET EMIN DEMIRKOL, is with Department Faculty Medicine of Çukurova University Faculty, Adana,Turkey, (e-mail: emindemirkol@gmail.com).

(iD https://orcid.org/0000-0003-3965-7360

ESEN YILDIRIM, is with Department of Electrical-Electronics Engineering, Adana Alparslan Türkeş Science and Technology University, Adana, Turkey, (e-mail: eyildirim@atu.edu.tr)

(iD https://orcid.org/0000-0002-4650-1164

LUT TAMAM, is with Department Faculty Medicine of Çukurova University Faculty, Adana,Turkey, (e-mail: 1tamam@gmail.com).

(iD https://orcid.org/0000-0002-9750-7531

Manuscript received October 11, 2019; accepted December 23, 2019. DOI: $10.17694 /$ bajece.631951

\section{INTRODUCTION}

$I^{\mathrm{N}}$ $\mathrm{N}$ ANY movement that occurs in the human body, electrical activity occurs in nerve cells in the brain. These activities are recorded with the help of sensors, and the recorded electrical data are called EEG. The frequency range of the EEG data is between $0.5-100 \mathrm{~Hz}$ and the amplitude is between 1 and $100 \mu \mathrm{V}$. EEG data are recorded with electrodes placed on the scalp according to the international 10-20 system.

Depression was considered by WHO to be the second most important health problem in 2020 that threatened people's lives [1]. Today, various scales are used in the diagnosis of depressive disorder in individuals. The application of various diagnostic scales used in the diagnosis of depression is based on the individual's declaration. In addition to the individual declaration, in recent studies, EEG signals have been used as a biomarker for the diagnosis of depression [2,3]. Also, EEG data are used in the diagnosis of depressive disorders as well as in investigating the effects of drugs used. In 2014, in the study of Erguzel et al. [1], EEG signs were used to determine the amount, level and effect of the drugs used in the treatment of major depressive disorders [1]. In the study, patients with depressive disorder and drug therapy were divided into two groups as responders and non-responders and EEG signals were collected. As a result of this procedure, the EEG data of the responding group and the non-responding group were classified with an accuracy rate of $85.45 \%$. Mallikkarjun et al. [4] studied the classification of EEG signals, which are considered to be one of the symptoms of depressive disorder and depressive symptoms of the patients with depressive disorder. In their study, EEG data of 240 patients with sleep problems and 60 patients who needed alcohol were classified and $88.32 \%$ accuracy rate was obtained. In the studies conducted recently, EEG signals of individuals diagnosed with Major Depressive Disorder are examined, and it is tried to obtain meaningful results from these signals $[5,6]$.

In this study, EEG signals were collected on a voluntary basis from the patients whose depressive disorder were diagnosed by using the scales applied in the Psychiatry Department of Çukurova University Medical Faculty clinically. These signals are collected by means of electrodes placed in accordance with the international $10 / 20$ system. The aim of this study was to determine whether the EEG data collected from the patients with depressive disorder in the eyes open and closed state could be differentiated from each other. 


\section{METHODS}

In the scope of this study, 30 patients diagnosed as having depressive disorder who were treated in Balcalı Hospital Psychiatry Department of Çukurova University Medicine Faculty were included in the study. In addition to EEG measurements, sociodemographic data form prepared by the researchers, Psychological Pain Scale and Beck Depression Scale were fulfilled by the participants.

\section{A. Psychological Pain Scale}

It is a five-point Likert-type self-report scale developed by Holden et al. Nine items of the scale assess the frequency of psychological pain and four items evaluate its intensity. Responses to the scale are in the range between never-always or strongly disagree-strongly agree. It was observed that the scale successfully differentiated suicide attempters and nonattempters [7]. A high score from the scale indicates that psychological pain is greater. In the Turkish validity and reliability study, Cronbach's alpha value was 0.98 [8].

\section{B. Beck Depression Scale}

It is a quartet Likert type self-report scale consisting of 21 items developed by Beck. The higher the score, the greater the severity of depression. In the Turkish validity and reliability study, Cronbach's alpha value was 0.80 [9].

\section{Collection of EEG Data}

EEG data from 30 patients with clinical depressive disorder were recorded with eyes open and closed. During the recording process, the patient was asked to keep his eyes closed and open for 1 minute according to the voice command. Figure 1 is a representation of recording process.

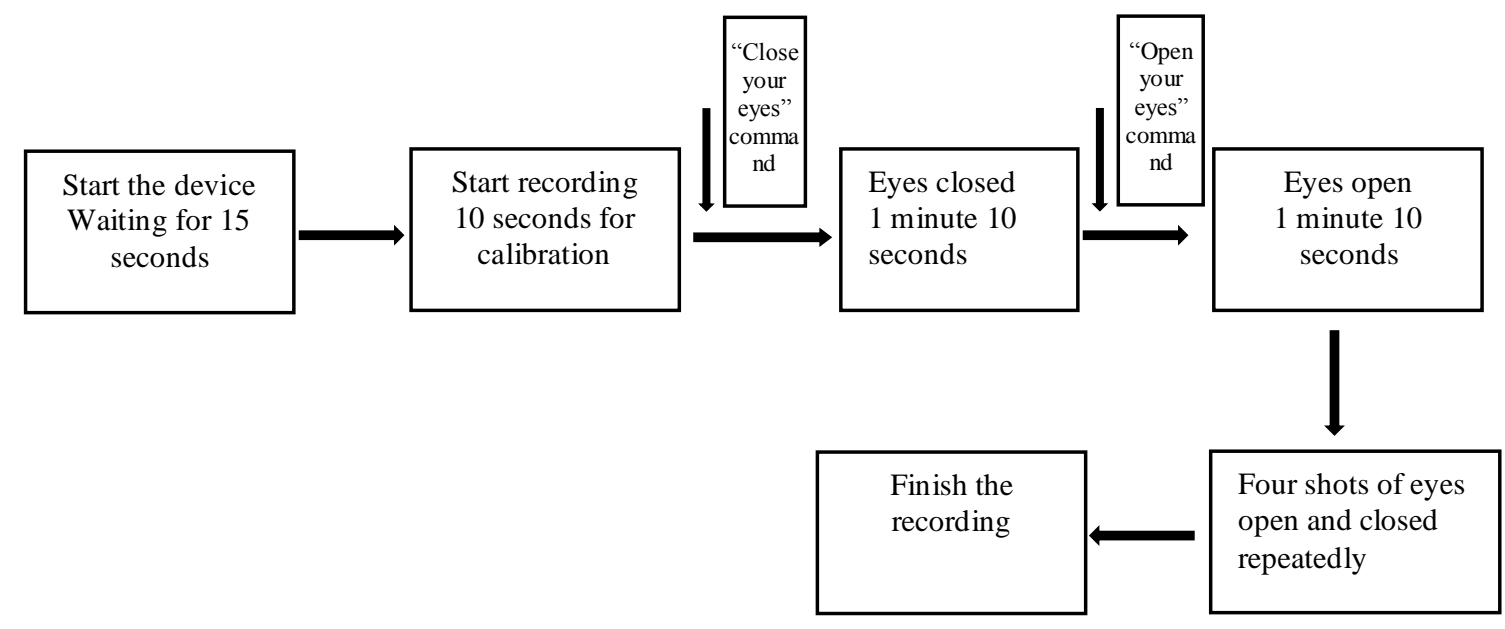

Figure 1. Representation of recording process.

Steps of the recording are given below:

1 - The device is activated with the first command and it is ensured that the patient and the project team attendant who will start the shooting are ready in 15 seconds.

2- The recording is started with the second command and it is waited for 10 seconds for calibration.

3- The eye closure command is given by voice as the third command and the subject is asked to close his eyes. At the same time, the moment when eye closed is enrolled by putting a marker on the recording by recording attendant.

4- Opening the eyes command was given 1 min 10 seconds after the third command. After this command, another marker was placed.

5- Steps 3 and 4 are repeated for 4 more times.

6- The recording process is finished.

In this setup, 1 minute 10 seconds recording time is given for each command and it is aimed to adapt the subject to the command in the closing or opening of the eye in the first 5 seconds. The last 5-second period was removed from the segments by aiming that the eye closed or open shooting times after each command were exactly 1 minute, the shifting of marker insertion times or eliminating the artifacts that could occur from the voice command in the subjects.

Before recordings, each subject was reminded that they should not move their hands, arms, legs and heads, as this causes noise on EEG signals during recordings. Environment was prepared according to the following:

1 - It has been paid attention that it is a quiet area to avoid distractions,

2- The subjects were provided to sit comfortably,

3 - It is ensured that the subjects look at a flat wall in such a way that there was no stimulus in front of them to distract their attention when the eye was open,

4- The subjects were asked to obey the commands that would require keeping their eyes open or closed during the shooting,

5- Experimenter acted in a way that does not make any distracting movement or sound during recordings,

EEG data were recorded with Emotiv EPOC which is a highquality wireless EEG device. Line noise was removed automatically by the device. Figure 2 shows the EEG recorder. 
Channels present in the device are; AF3, F7, F3, FC5, T7, P7, $\mathrm{O} 1, \mathrm{O} 2, \mathrm{P} 8, \mathrm{~T} 8, \mathrm{FC} 6, \mathrm{~F} 4, \mathrm{~F} 8$, AF42. Sampling frequency is $128 \mathrm{~Hz}$ and a bandpass filter is applied by the device to keep the frequency content between 0.2 and $45 \mathrm{~Hz}$.

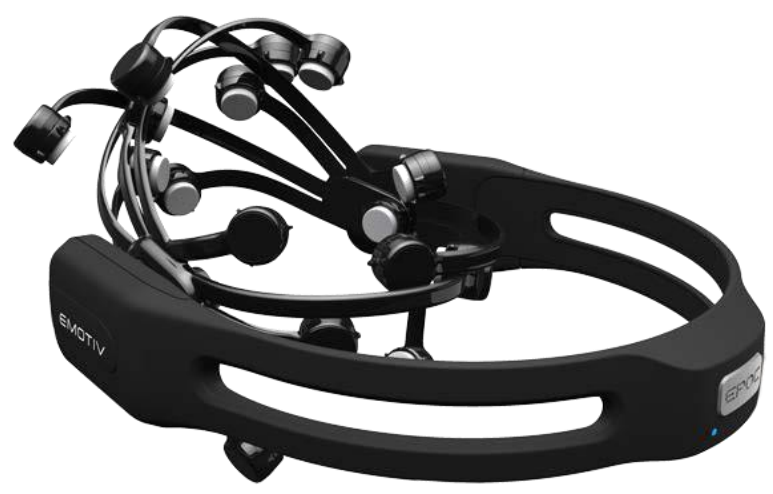

Figure 2 Emotive EPOC EEG Device

The device is placed on the skull in accordance with the international 10-20 system, and the electrodes are located As shown in Figure 3.

\section{Feature Extraction}

The collected $7 \mathrm{~min}$ and 10 secs raw EEG data were divided into segments. A total of six 1-minute EEG data were obtained, 3 closed eyes and 3 open eyes, for each subject. Features are extracted by employing Continuous Wavelet Transform (CWT). The wavelet model used for CWT is the Morlet wavelet. total energy and energies in theta $(3-7 \mathrm{~Hz})$, alpha $(8-12 \mathrm{~Hz})$, beta $(13-29 \mathrm{~Hz})$ and gamma $(30-45 \mathrm{~Hz})$ bands obtained for each channel. The total number of features is 14 channels x 5 energy values $=70$. Two-seconds sliding windows with $50 \%$ overlap windows were used for feature extraction.

As a result, a feature matrix of $179 \times 70$ was obtained for each eye state (open/closed) from each subject.

\section{E. Classification with $k N N$ Algorithm}

In this study, k-Nearest Neighbor (kNN) algorithm is used to classify EEG signals $[10,11,12]$. K-Nearest Neighborhood $(\mathrm{kNN})$ classification algorithm is a theorem that works according to the distance value between classes and the nearest neighbor value to be selected. According to

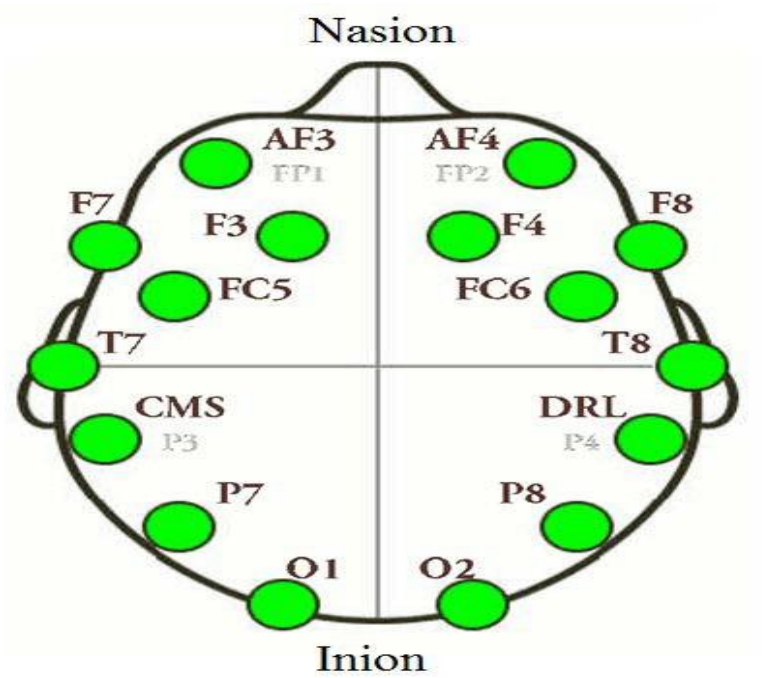

Figure 3 Placement of electrodes according to 10-20 system for EEG recording (top view)

this classification algorithm, $\mathrm{k}$ points are selected to be closest to the data. The classification process is made according to this selected value. The small value of $k$ helps to collect the sample with the highest similarity rate in the same class while increasing $\mathrm{k}$ value can cause different classification results by including dissimilar data in the same class [13]. Therefore, one of the methods used to select the $\mathrm{k}$ value is to compare the classification results for various $\mathrm{k}$ values, although it takes time. For example, in the study of Özer and Amasya, the classification success obtained when the classification of the data was made according to the 1-nearest neighbor acquired better results than the classifications of $3,5,7,9$ and 10-nearest neighboring [14].

\section{RESULTS}

In this study, the feature matrices obtained from the collected EEG data were created independently from the subject and these matrices were classified according to their neighbor values closest to $1,3,5$ in the $\mathrm{kNN}$ algorithm and the results are given in Table 1.

TABLE I

CLASSIFICATION RESULTS

\begin{tabular}{|l|l|l|l|l|l|l|}
\hline kNN & TP Rate & FP Rate & Precision & Recall & F-Measure & ROC Area \\
\hline 1 & 0.913 & 0.087 & 0.913 & 0.913 & 0.913 & 0.913 \\
\hline 3 & 0.897 & 0.103 & 0.897 & 0.897 & 0.897 & 0.951 \\
\hline 5 & 0.892 & 0.108 & 0.892 & 0.892 & 0.892 & 0.957 \\
\hline
\end{tabular}

EEG recordings collected from 30 patients with clinically depressive disorder for eyes open/closed states were classified with $91.3 \%, 89.7 \%$ and $89.2 \%$ accuracy for $\mathrm{k}=1$, 3 and 5, respectively, according to the $\mathrm{kNN}$ classification algorithm. The highest success was obtained for $\mathrm{k}=1$. As the results show, the EEG signals recorded from patients with depressive disorder as their eyes were open or closed can be distinguished from each other with a very high accuracy. In future studies, the differentiation accuracies of the EEG signals collected from individuals with clinical depressive disorder and the control group that have the same demographic structure will be examined. 


\section{ACKNOWLEDGMENT}

This study was supported by the Scientific Research Projects Unit of Adana Alparslan Turkes Science and Technology University. Research Grant No.: 18103031

\section{REFERENCES}

[1] T. Erguzel, S. Ozekes, A. Bayram and N. Tarhan, "Classification of major depressive disorder subjects using Pre-rTMS electroencephalography data with support vector machine approach," 2014 Science and Information Conference, London, 2014, pp. 410-414.

[2 ] S. A. Akar, S. Kara, S. Agambayev and V. Bilgiç, "Nonlinear analysis of EEG in major depression with fractal dimensions," 2015 37th Annual International Conference of the IEEE Engineering in Medicine and Biology Society (EMBC), Milan, 2015, pp. 7410-7413.

[3] W. Mumtaz, A. S. Malik, S. S. A. Ali and M. A. M. Yasin, "P300 intensities and latencies for major depressive disorder detection," 2015 IEEE International Conference on Signal and Image Processing Applications (ICSIPA), Kuala Lumpur, 2015, pp. 542-545.

[4] H. M. Mallikarjun and H. N. Suresh, "Depression level prediction using EEG signal processing," 2014 International Conference on Contemporary Computing and Informatics (IC3I), Mysore, 2014, pp. 928-933.

[5] A. M. Al-Kaysi, A. Al-Ani, C. K. Loo, M. Breakspear and T. W. Boonstra, "Predicting brain stimulation treatment outcomes of depressed patients through the classification of EEG oscillations," 2016 38th Annual International Conference of the IEEE Engineering in Medicine and Biology Society (EMBC), Orlando, FL, 2016, pp. 52665269.

[6] Y. Mohan, S. S. Chee, D. K. P. Xin and L. P. Foong, "Artificial neural network for classification of depressive and normal in EEG," 2016 IEEE EMBS Conference on Biomedical Engineering and Sciences (IECBES), Kuala Lumpur, 2016, pp. 286-290

[7] Holden, R. R., Mehta, K., Cunningham, E. J., \& McLeod, L. D. (2001). "Development and preliminary validation of a scale of psychache". Canadian Journal of Behavioural Science/Revue Canadienne Des Sciences Du Comportement, 33(4), 224.

[8] Demirkol, M., Güleç, H., Çakmak, S., Namlı, Z., Güleç, M., Güçlü, N., \& Tamam, L. (2018). "Reliability and validity study of the Turkish Version of the Psychache Scale". Anatolian Journal of Psychiatry, 19(1), 14-20.

[9] Beck, A. T., \& Steer, R. A. (1984)." Internal consistencies of the original and revised Beck Depression Inventory". Journal of Clinical Psychology, 40(6), 1365-1367.

[10] Qu , H. \& Gotman, J. A, "Patient-Specific Algorithm for the Detection of Seizure Onset in Long-Term EEG Monitoring: Possible Use as a Warning Device", IEEE Transactions on Biomedical Engineering, 44, 115-122p, 1997.

[11] Oliveria, I., Grigori, O., Guimaraes, N., "EEG Signal Analysis for Silent Visual Reading Classification", International Journal Of Circuits, Systems And Signal Processing, Issue 3, Volume 3, 2009:119-126p.

[12] Murugappan, M., "Human emotion classification using wavelet transform and KNN", Pattern Analysis and Intelligent Robotics (ICPAIR) International Conference on, (Volume:1 ), 28-29 June 2011, $148-153 \mathrm{p}$.

[13] Mitchell, T., 1997, "Machine Learning”, McGraw Hill, 1997.

[14] Özer, Z.B., ve Amasyalı, M.F., "Meta Öğrenme ile KNN Parametre Seçimi”., 21. Sinyal İşleme Ve Uygulamaları Konferansı, 2013.

\section{BIOGRAPHIES}

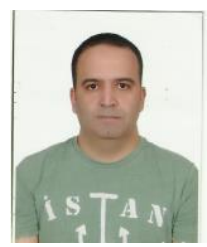

Server Göksel ERALDEMIR $\mathrm{He}$ received the Bachelor degree in Computer Education and Instructional Technology from the University of OMU, Turkey, in 2002. Then he worked computer teacher in Reyhanlı high school from 2002 to 2009 and After in late 2009 he started to work as a lecturer at the university. Then he received Masters Degree in electrical and electronics engineering from the University Mustafa Kemal, in 2014. In 2019, I started my doctoral education in the Department of Electrical and Electronics Engineering, Institute of Science and Technology, Adana Alparslan Türkeş University of Science and Technology.

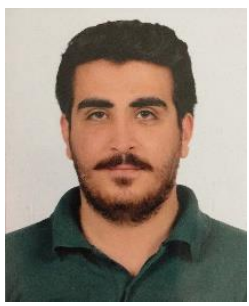

Ümit KILIÇ was born in 1992, in Adana, Turkey. He received the BSc degree in Computer Engineering from Çukurova University and the MSc degree in Nanotechnology and Engineering Sciences from Adana Alparslan Türkeş Science and Technolgy University in 2016 and 2019, respectively. He is currently a PhD student in the Department of ElectricalElectronics Engineering in Adana Alparslan Türkeş Science and Technology University. He also works as a Research Assistant in the Department of Computer Engineering at the same university. His research interests are Data Mining, Artificial Intelligence, and their applications. He can be reachedviaukilic@atu.edu.tr.

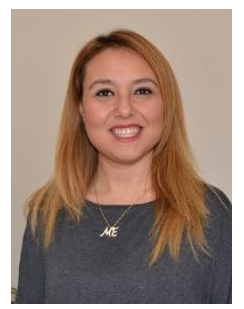

Mümine KAYA KELEŞ was born in 1986, in Adana, Turkey. She received the BSc and MSc degrees in Computer Engineering from Çukurova University, Adana, Turkey, in 2009 and 2011, respectively. She received $\mathrm{PhD}$ degree in Computer Sciences Division of Electrical and Electronics Engineering from Çukurova University, Adana, Turkey. She is currently Assistant Professor in the Department of Computer Engineering at the Adana Alparslan Türkeş Science and Technology University. Her research interests include Data Mining, Distance Education, Text Mining, Plagiarism Detection, Information Retrieval, Text/Document Processing, Web Mining, Database Management Systems, Natural Language Processing, and their applications. Contact her at mkaya@atu.edu.tr. Her ORCID ID is https://orcid.org/0000-0001-8414-1713.

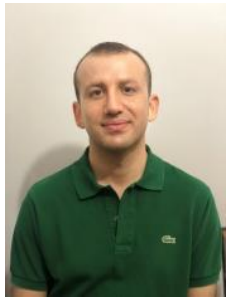

Mehmet Emin DEMIRKOL: Mehmet Emin Demirkol graduated from Çukurova University Faculty of Medicine in 2010. Between 2011 and 2015, he received psychiatry specialty training at the same university. He is still working as an instructor at Çukurova University Faculty of Medicine, Department of Psychiatry. He investigated the relationship between suicide attempts and 
psychache and alexithymia in patients with schizophrenia. $\mathrm{He}$ has studies about bariatric surgery patients and the efficacy of light therapy in depressive patients.

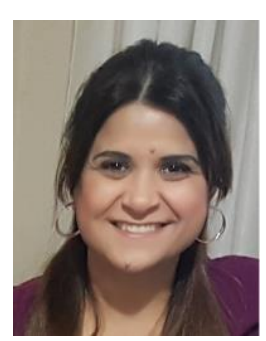

Esen Yildirim, received the B.S. degree in Electrical and Electronics Engineering from Cukurova University, Adana, Turkey, in 1997, and the M.S. and $\mathrm{Ph} . \mathrm{D}$. degrees in Electrical Engineering from University of Southern California (USC), Los Angeles, in 2000 and 2006, respectively. She is currently an Associate Professor of Electrical and Electronic Engineering, at Adana Alparslan Türkeş Science and Technology University, Adana, Turkey. Her general research interests include biomedical signal processing, epileptic seizure detection, functional connectivity, learning methods, and emotion recognition from physiological signals.

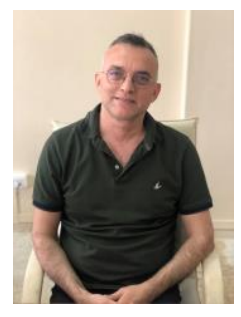

Lut TAMAM: Professor Doctor Lut Tamam has been working in the field of psychiatry for 25 years and is head of the Department of Psychiatry of Çukurova University Faculty of Medicine for 8 years. He has studies about comorbidity in bipolar disorder, impulse control disorders, attention deficit hyperactivity disorder, light therapy, electroconvulsive therapy, obsessive compulsive disorder, and major depressive disorder. He is also the editor of the journal Current Approaches in Psychiatry. 